\title{
Speed Up-Greedy Perimeter Stateless Routing Protocol for Wireless Sensor Networks (SU-GPSR)
}

\author{
Yan Sun \\ School of Electronic Engineering \\ and Computer Science \\ Queen Mary University of London \\ London, UK \\ yan.sun@qmul.ac.uk
}

\author{
Junpeng Guo \\ School of Electronic Engineering \\ and Computer Science \\ Queen Mary University of London \\ London, UK \\ j.guo@se13.qmul.ac.uk
}

\author{
Yuhui Yao \\ School of Electronic Engineering \\ and Computer Science \\ Queen Mary University of London \\ London, UK \\ yuhui.yao@qmul.ac.uk
}

\begin{abstract}
This paper proposes a position-based routing scheme called Speed Up-Greedy Perimeter Stateless Routing Protocol for Wireless Sensor Networks (SU-GPSR). The speed up mode works together with greedy mode to provide a solution for data transmission in WSN. Unlike other variants of greedy perimeter stateless routing protocols, SU-GPSR not only embraces both still nodes and mobile nodes in one unified scheme, but provide a complete energy consumption consideration when evaluating the potential next hop. The simulation results reveal that the SU-GPSR outperforms EGPSR and FA-GPSR from various aspects in both dense and loose network size.
\end{abstract}

Keywords-WSN; position; greedy; energy harvesting; routing; mobility;

\section{INTRODUCTION}

Wireless Sensor Network (WSN), as a network with a collective of sensor devices distributed within a certain geography area, was firstly proposed for serving military purposes in the early 20th Century. Along with the fast evolution of sensing technologies and chipsets since 1980s, WSN's applicable scenarios soon spread over environmental monitoring, vehicle tracking, inventory managing and many other more [1]. Further accelerated by the concept of Internet of Things (IoT), WSN is believed to be one of the essential networks benefiting the common welfare on earth. Introducing the WSN into habitat monitoring scenarios bear many advantages, such as rapid deployment of wireless devises for data collection, smooth integration of various type of monitoring data, flexible network structure for data communication under dynamic circumstances, etc [2]. The mixture of location fixed sensor nodes and mobile nodes in habitat monitoring environment brings new challenges when constructing the WSN. Especially, considering the data forwarding mechanism in network layer, how to design an effective routing strategy to face the dynamically changing network topology has been one of the hot research topics for decades. Various constrains, such as short radio transmission range, limited energy supply, node mobility, network size, have to be taken into consideration during the scheme design.

Along with the availability of tiny, inexpensive, low-power consumption GPS receivers, the position based routing solutions started to gain more attentions. Sensors with geographic information facilitate the environmental monitoring activities greatly. Many researchers looked into the technical details to seek the advanced solutions. Ivan in his survey work [3] listed a table of most popular position-based routing methods adopted in WSN for key feature comparison. Authors in [4] has pointed out that compared to the topology-based routing protocols which do not make use of geographic information in the routing decision, position-based routing protocols are more scalable. Among them which have the characters of loop-free, localization and single path, the two-mode mechanism (greedy mode and recovery mode) are commonly adopted [5-11]. In this work, we first analyze the most recent greedy/recovery mode based routing protocols in WSN. With better understanding of the current limitations, we proposed a Speed Up-Greedy Perimeter Stateless Routing Protocol (SU-GPSR) which aims to reduce the end-toend delay during data forwarding process for habitat monitoring WSN. The main contributions of this work are listed below:

- $\quad$ The recovery mode is replaced by a newly designed Speed-Up mode in SU-GPSR to reduce the number of hops along the transmission route caused by the operations in traditional greedy/recovery mode.

- $\quad$ SU-GPSR automatically adapts the routing strategy according to the network density. It is in favour of moving nodes when nearby still nodes are not reachable.

- A hop by hop delay together with remaining lifetime observation mechanism is constructed to provide real-time evaluation of potential delay introduced by next hop selection algorithm.

- Sensor nodes' energy harvesting capability modelling is adopted as an additional contributor in next hop selection algorithm

The detailed SU-GPSR design is addressed in the section right after related work below.

\section{RELATED WORK}

The general concept of greedy/recovery mode in positionbased routing protocols is to categorize the data forwarding operations into two set of strategies. In the greedy mode, the sensor node is able to advance the data towards the final destination. Quite a few work [6-11] have been working on possible solutions about how to select the most suitable next hop towards the final destination, given different considerations, such as geographic distance between each pair nodes or the line 
connecting the source and destination nodes. If a node cannot find the next hop to advance the data in greedy mode, the node will transfer into recovery mode. Again, various actions have been proposed in the recovery mode by researchers but with the same intension, which is to allow the nodes who carry the data can eventually transfer back to the greedy mode for further data forwarding towards the final destination. Greedy Perimeter Stateless Routing Protocol (GPSR) [12], proposed by Karp and Kung in 2000, is a well-known geographical-based routing protocol for Mobile Ad Hoc Networks (MANET). GPSR allows nodes in greedy mode to forward the packet through the shortest path between source and destination. The recovery mode makes use of the perimeter of a network area to forward the data by applying a simple right-hand rule. Divisional Perimeter (DP) is proposed in [13] to improve the GPSR performance by employing both right-hand and left-hand rules. Buffering Zone Greedy Forwarding Strategy (BZGFS) [14] is another variation of original GPSR which deprioritizes the nodes at the transmission range edges when recovery mode action is taken. In the version of GPSR-MA (Movement Aware GPSR) [15], the mobility handling capability is added into the conventional GPSR. Ferry-Assisted GPSR (FA-GPSR) [16] was proposed in 2014 to further explore the cases when a patrolling node is involved. The key features of FA-GPSR are highlighted below.

- A Patrol Seeking mode is added as the third mode working in case of the recovery mode fails.

- Unlike previous work which let the node to drop the packet if recovery mode is not successful, nodes in Patrol Seeking mode will forward the data towards an appropriate ferry node.

- $\quad$ The ferry node, moving along the predefined path, will catch the packets until reaching a position which is closest to the final destination. Then the ferry node will forward the data towards the final destination via the selected next hop.

- The Patrol Seeking mode will just be entered once. If another recovery mode fails again, the packet will be dropped

NS-3 simulation platform was adopted in [16] for FA-GPSR performance evaluation, comparing with GPSR, GPSR-DP and GPSR-BZGFS. The results illustrate that with the assistance of ferry nodes, FA-GPSR improves the average packet delivery rate greatly (up to about $50 \%$ ) along with the number of nodes increasing from 50 to 200. The high end-to-end delay in FAGPSR is one of the shortcomings due to buffered packets in the ferry nodes and moving speed for packet delivery. The simulation results also reveal that all four GPSR variants suffer high hop counts regardless of the network density. Such big number of hops (between 40-60) for delivering every packet will introduce great risks of further delay (due to the buffering and queuing at each hop), high packet collision (due to the number of nodes involving in transmission) and severe interferences (due to the more concurrent co-channel transmissions).

A new Enhanced Greedy Perimeter Stateless Routing (EGPSR) was proposed in work [17] recently. The main contribution of this work is to divide a forward region into a few sub-regions with equal area. The sub-region selection is carried out based on the remaining energy of the neighbour nodes in each sub-region. The sub-region with the highest remaining average node energy will be selected. Then the next forwarding node will be selected from this sub-region according to the defined node probability transmission model. The performance of EGPSR was evaluated via simulation on Matlab platform. Compared to GPSR and IGPSR (Improved GPSR), EGPSR outperformed in the aspect of high packet delivering number and low network energy consumption.

However, the EGPSR design still overlooked certain issues. Firstly, only the node's current remaining energy is considered in EGPSR. In actual WSN, both receiving and transmitting data require energy as well. Whether or not the current remaining energy at each node can fulfil the forwarding tasks is missing in EGPSR. If the answer is false, the next hop selection might result in packet lost. Secondly, both IGPSR and EGPSR scheme aim at the improvement when nodes are in greedy mode. There is no explanation about what are the actions once the nodes fall into recovery mode. Furthermore, no mobile nodes are taken into consideration in EGPSR.

In this work, SU-GPSR is proposed to deal with the remaining issues mentioned above with features below:

- A Speed Up mode is created to replace recovery mode. If the packet is carried by a ferry node, the transmission is in Speed Up mode. Otherwise the transmission is in Greedy mode.

- A unified next hop selection criteria is designed to provide an integrated solution for both Greedy and Speed-Up modes, which serves the less-hop count route selecting intension adaptively based on the nodes' density.

- The energy consumption for receiving and transmitting packet at the potential next hop is taken into account for measuring the future remaining energy. Furthermore, the harvested energy is considered as well if the nodes are equipped with energy harvesting capability.

- The remaining packet lifetime contributes to the routing strategy decision at each node, given overall network resource utilization. The mobile nodes are preferable in order to reduce the total number of hop counts for each packet transmission, as long as the packet delay satisfies the QoS requirements.

Overall, in SU-GPSR, the still nodes and ferry nodes are treated equally when next hop selection algorithm is running. The two modes are no longer referring to each node, but to the transmission. The overall design target of SU-GPSR is to reduce the total number of hops along the route from source to the destination. The next section gives scheme details. 


\section{SU-GPSR SCHEME DESIGN}

\section{A. Forward Region and Sub-Region}

SU-GPSR adopts the same principle as addressed in EGPSR for forwarding region division. However SU-GPSR considers node $i$ 's future remaining energy $E^{\prime}{ }_{i}$ instead of the present remaining energy when measuring the average energy of all the nodes in each sub-region.

$$
E_{i}^{\prime}=E_{i}-R E \times R B-S E \times S B \times d_{i j}+H E_{i}
$$

Where $E_{i}$ is node $i$ 's present remaining energy, $R E$ and $S E$ denote the energy consumption for receiving and transmitting each bit respectively, $S B$ and $R B$ indicate the size in bit of the transmitted and received packets. $d_{i j}$ denotes the distance between node $i$ and neighbour node $j . H E_{i}$ is the harvested energy at node $i$ if harvesting is enabled. Referring to work [18], we have

$$
\begin{gathered}
H E_{i}=E_{i} \times \rho \times\left(-e^{\frac{-k_{i}}{n}}+1\right) \\
k_{i}=\frac{v_{i}^{p v} \times I_{i}^{p v}}{C_{i}}
\end{gathered}
$$

Where $\rho$ is the energy harvesting rate, $v_{i}^{p v}$ and $I_{i}^{p v}$ are the output voltage of the Photovoltaic panel and output current of node $i . C_{i}$ is the real-time battery measurement at node $i$.

Then we have the average energy of each sub-region as

$$
\overline{E_{l r}}=\frac{\sum_{j=1}^{N_{i r}} E_{j}}{N_{i r}}
$$

Where $N_{i r}$ is the total number of nodes in the sub-region $r$.

Fig. 1 is given blow to illustrate the forwarding region division. $N_{i}$ is the current node, $N_{j}$ is candidate of next hop node and $N_{s}$ is the sink node.

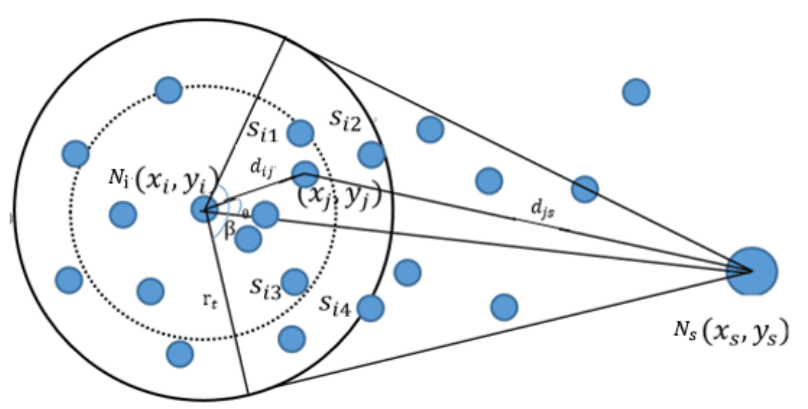

Fig. 1 The Division of the Forward Region [17]

The pseudo code of sub-region selection algorithm is shown in Algorithm 1.

1. Input Neighhop_List, WaitList

2. Insert element in WaitList to Neighhop_List
3. For $\mathrm{i}<$ NeighHop_List length do

4. Get the location of next hop $\mathrm{j}$

5. If dis_to_nexthop $>r_{T}$

6. Store $\mathrm{j}$ into WaitList, $\mathrm{n}=0$

7. Else

8. Call DivedeSubForwardRegion(List

NeighHop_list)

9. $\quad$ End if

10. Call SelectNextHop(List NeighHop_subregion)

11. End for

12. Judge $\mathrm{n}$ whether still in NeighHop_list, if not $\mathrm{n}=0$

13. Output $n$

14. Function DivdeSubForwardRegion(List NeighHop_list)

15. Traverse the entire NeighHop list

16. Divide these NeighHop into different regions with equal area

17. Calculate the average energy of each sub-region $\bar{E}_{\mathrm{ir}}$

18. Select the biggest the $\bar{E}_{\text {ir }}$

19. Return the corresponding list of NeighHop_Subregion with biggest $\bar{E}_{\mathrm{ir}}$

20. End Function

21. Function SelectNextHop(List NeighHop_subregion)

22. Traverse the entire list

23. Calculate the probability $\mathrm{p}$ of each possible nexthop according to formula(5)

24. Select biggest $\mathrm{p}$

25. Return the corresponding nexthop $\mathrm{n}$ with biggest $\mathrm{p}$

26. End Function

Algorithm 1 Pseudo Code of Sub-region Selection Algorithm

In the Algorithm 1, line 1-13 is the main function to select the sub-region which is with the biggest remaining energy on the account of extending the network lifetime. If the mobile node is beyond the transmission radius, it will be removed from the next hop candidate list and restored in the waiting list for future use. Line 14-20 is the function to divide the total forwarding area into $\mathrm{r}$ sub-regions with same areas, for instance $S_{i 1}, S_{i 2}, S_{i 3}$ and $S_{i 4}$ as shown in in Fig. 1. Line 21-26 is the function to determine the final next hop within the chosen sub-region based on the strategy. Details will be addressed in the next section.

\section{B. Next hop Selection with Node Probability}

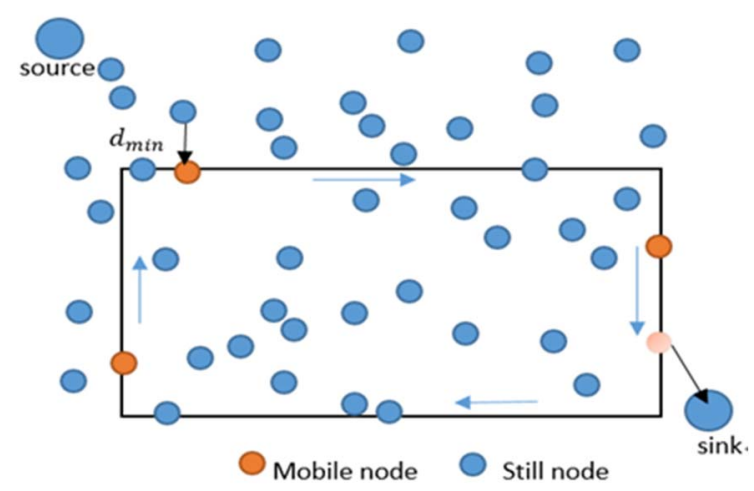

Fig. 2 Data Transmission Scenario in habitat monitoring WSN 
Taken a habitat monitoring scenario as an example, there are a few still nodes located between the far apart source and sink node in WSN, as illustrated in Fig. 2. There are also mobile nodes, such as sensors mounted on vehicles, moving along the pre-defined route.

In SU-GPSR, the design of next hop selection algorithm intends to apply the same criteria to still and moving neighbor nodes. The outcome of the node selection algorithm determines which mode the next transmission belongs to. If a still node is chosen, the transmission is in Greedy mode. Otherwise, the transmission is in Speed-Up mode. Similar to EGPSR, the next hop selection algorithm is based on the probability of each node in the selected sub-region, as shown in equation (5)-(7) below.

$$
\begin{aligned}
& \mathrm{P}\left(N_{j}, E_{j}, d_{j s}, \theta, \alpha, t_{d i}\right)= \\
& \left\{\begin{array}{cc}
\frac{\alpha \times E_{j}}{\sum_{j=0}^{N_{i r}} E_{j}} \times \frac{N_{j}}{\sum_{j=0}^{N_{i r} N_{j}}} \times\left(1-\left(\frac{d_{j s}}{\sum_{j=1}^{N_{i r}} d_{j s}}\right)\right) \times \cos \theta \times F,\left|N_{i r}\right|>2 \\
1, & \left|N_{i r}\right|=1 \\
0, & \text { else }
\end{array}\right.
\end{aligned}
$$

Where $N_{j}$ is the total number of candidate of next hop, $F$ is the weight influencing the selection of still node or mobile node as shown in (6), $\alpha$ is the power coefficient determined by (7)

$$
\begin{gathered}
F=(\mathrm{M}+1)^{\left[\frac{T_{L}-\left(T_{\text {now }}-T_{\text {gene }}\right)}{T_{L}}-\frac{1}{2}\right]} \\
\alpha=(1+k)^{1+\rho}
\end{gathered}
$$

Where $M$ is the factor indicating the candidate of next hop is still node or mobile node set by $(8), T_{\text {now }}$ is the observation time at node $i, T_{\text {gene }}$ is the time of generating this packet, $\mathrm{k}$ is the Boolean value set by (9)

$$
\mathrm{M}=\left\{\begin{array}{c}
0, \text { if still node } \\
1, \text { if mobile node }
\end{array}\right.
$$

$$
k=\left\{\begin{array}{c}
1, \quad \text { next hop with harvesting } \\
0, \quad \text { next hop without harvesting }
\end{array}\right.
$$

$T_{L}$ is maximum allowed packet lifetime in the network defined in (10). Based on Chebyshev inequality to determine the maximum packet lifetime $\mathrm{T}_{L}$ to achieve the $90 \%$ mobile nodes could arrive to the nearby of sink node.

$$
\mathrm{P}\left(\left|\mathrm{T}_{L}-\frac{d}{2 E(v)}\right|<\frac{d}{\sqrt{12 D(v)}}\right) \geq 0.9
$$

Where $d$ is the perimeter of the pre-defined moving path, the positions of the mobile nodes are uniformly distributed.

The equation (5) design reflects the core intension of SUGPSR which is to treat all the potential next hop equally, no matter still nodes or mobile nodes, with factors of energy capability, distance toward the final destination, and remaining lifetime for data forwarding

\section{SimUlation AND EVALUATION}

To evaluate the performance of SU-GPSR design, OPNET is used as the simulation platform. The scenarios consist of still and mobile notes which allows the full function of SU-GPSR to be testified. EGPSR and FA-GPSR are implemented in the platform as well for comparison. For every scenario, the configuration is the same for SU-GPSR, FA-GPSR and EGPSR schemes. Each scenario is repeated 10 times with different random seeds. Each call lasts 8 minutes. For clarity, only the mean values are shown in this section.

\section{A. Simulation Scenario and Configurations}

The simulation runs within a $1000 \mathrm{~m} * 1000 \mathrm{~m}$ area. The source and destination nodes are located at the opposite corners with fixed location as illustrated in Fig. 3. The total number of nodes is increasing from 150 to 200 to give different network density. They are randomly distributed within the area. The square line is pre-defined trajectory which mobile nodes will follow during the simulation. For each scenario there are five, seven and nine mobile nodes respectively. They are moving clockwise along the line with random speed within the range $[30 \mathrm{~km} / \mathrm{h}, 60 \mathrm{~km} / \mathrm{h}]$.

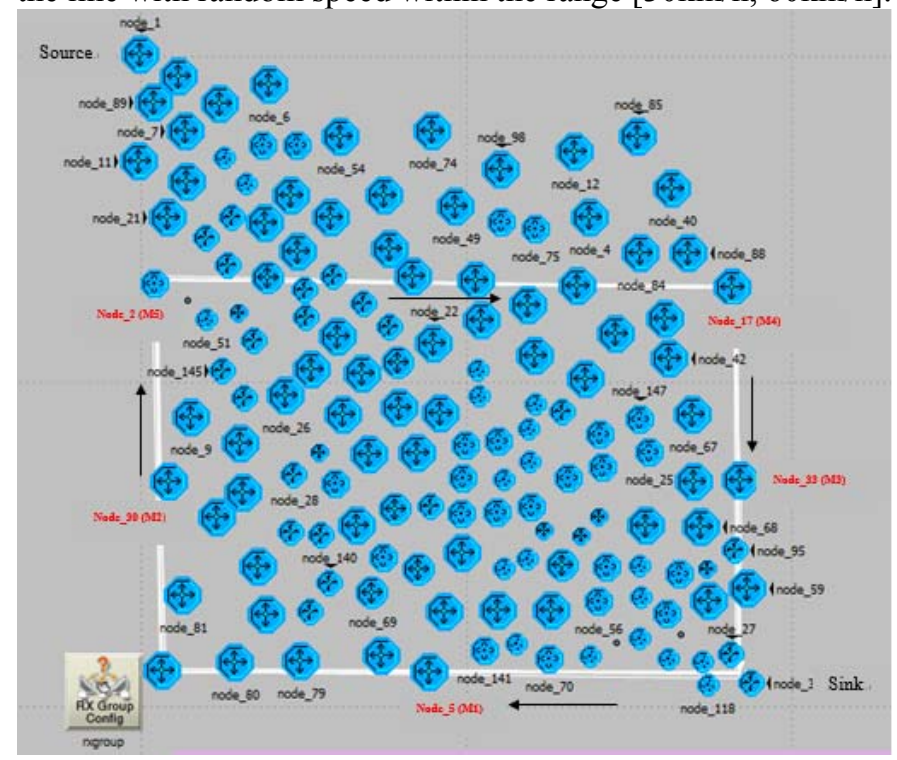

Fig. 3 Simulaiton Scenario Example with Three Moible Nodes

TABLE 1 lists the parameters' setting for all the simulations.

TABLE 1 PARAMETERS CONFIGURATION

\begin{tabular}{|c|c|}
\hline Parameter & Value \\
\hline$\rho$ (Solar Energy Harvesting Rate) [18] & $97 \%$ \\
\hline RE (Receiving Energy, J/bit) & $50 *(1 \mathrm{e}-9)$ \\
\hline SE (Sending Energy, J/bit) & $50 *(1 \mathrm{e}-9)$ \\
\hline Harvesting Node Ratio & $10 \%$ \\
\hline Data Generation Rate (packets/second) & 1 \\
\hline Node Transmission Range (m) & 75 \\
\hline
\end{tabular}

\section{B. Performance Analysis}

Firstly the network life time is analysed among three routing schemes while the network size grows from 150 to 200 . The lifetime is measured as the simulation duration till the first node 
dies due to the energy drain out. The simulation terminates after 8 minutes by force.

Bars in Fig.4 indicate the network lifetime of each simulation scenario. With more nodes in the network, the longer network lifetime they can experience. When the node density reaches 1.14, both SU-GPSR and FA-GPSR can last till the end of simulation. Because both schemes consider the energy factor when selecting the next hop and there are more nodes existing in the network to even out the energy consumption for forwarding the packets. EGPSR performs the worst among three routing protocols along all network scales.

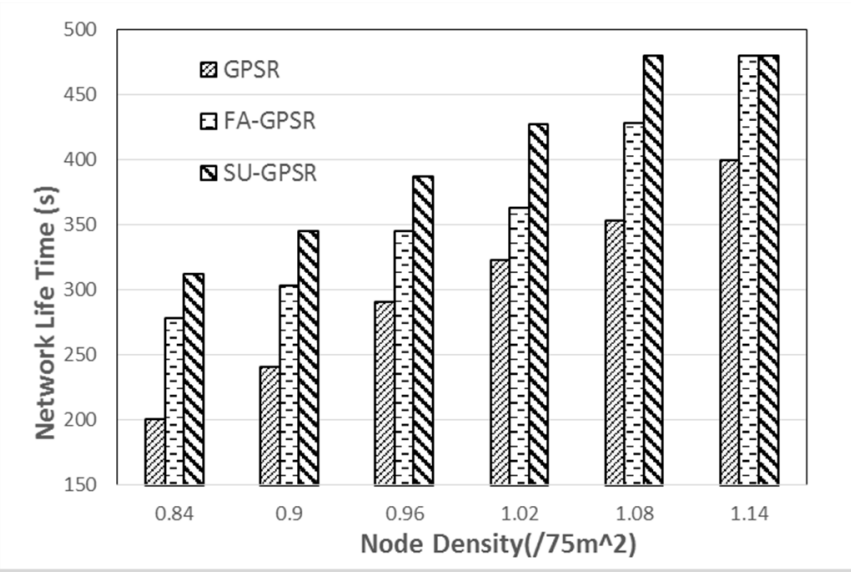

Fig. 4 Network Life Time with Various Network Size

The end-to-end delay is discussed in Fig. 5 for SU-GPSR and FA-GPSR with five, seven and nine mobile nodes. Both protocols conduct long delay when the network density is low. In the simulation, the low packet generation rate is set to guarantee that the queuing delay in the buffer is not considered in this work. Only transmission delay (light speed between still nodes and mobile speed by mobile nodes) are observed in the simulation. When less still nodes are available in the area, SUGPSR intends to select the available mobile nodes to reduce the total number of hops, which leads to higher end-to-end delay. As the number of mobile nodes stay the same but more still nodes are available, the end-to-end delay is reduced dramatically. Overall the SU-GPSR performs with higher end-to-end delay compared to FA-GPSR due to the mobile nodes' involvement. However, real-time remaining packet lifetime consideration in SU-GPSR routing strategy allows the less hops achievement without risking the packet invalidation.

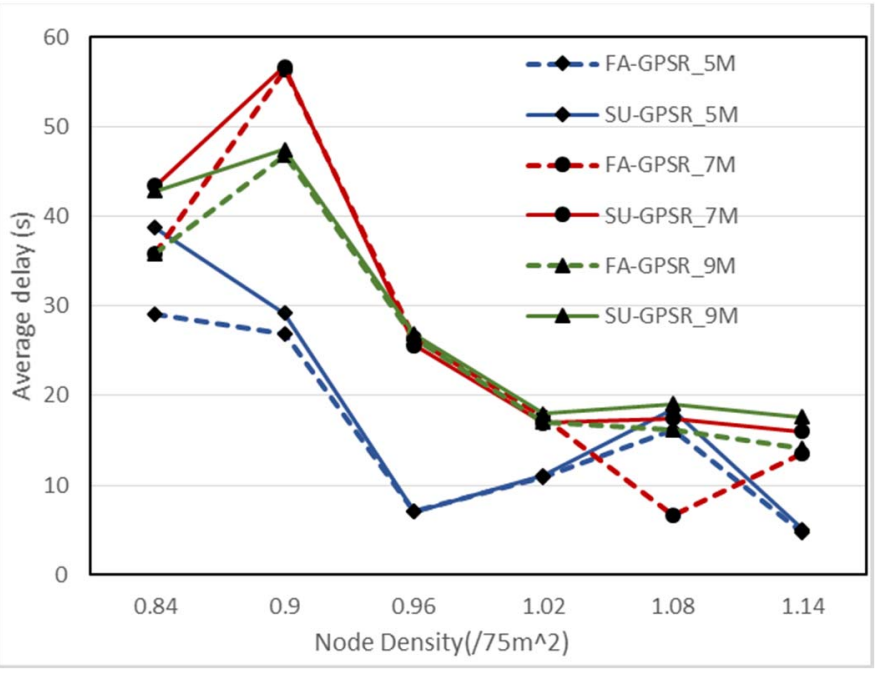

Fig. 5 Average End-to-End Delay with various Node Density

Both FA-GPSR and SU-GPSR consider mobile nodes when advancing the packet to destination. The difference between them is FA-GPSR only choose the available ferry node if recovery mode fails, while SU-GPSR treats the mobile nodes and still nodes equally when selecting the next hop. In order to reduce the total number of hops along the selected route, SUGPSR prefers the mobile node even if there are other still candidates, given the estimated transmission time is within the packet lifetime allowance. The average hop counts is shown in Fig. 6. SU-GPSR has outperformed FA-GPSR for all scenarios.

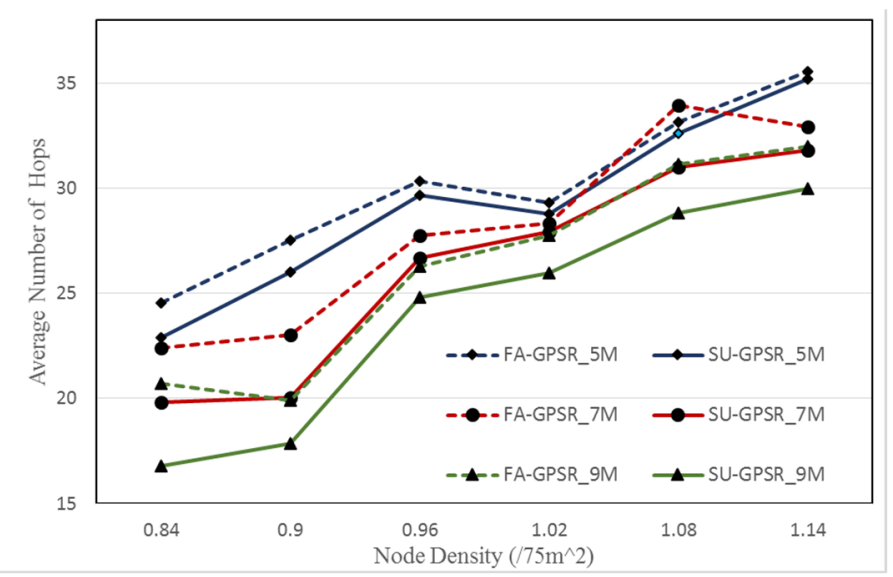

Fig. 6 Average Hop Counts 


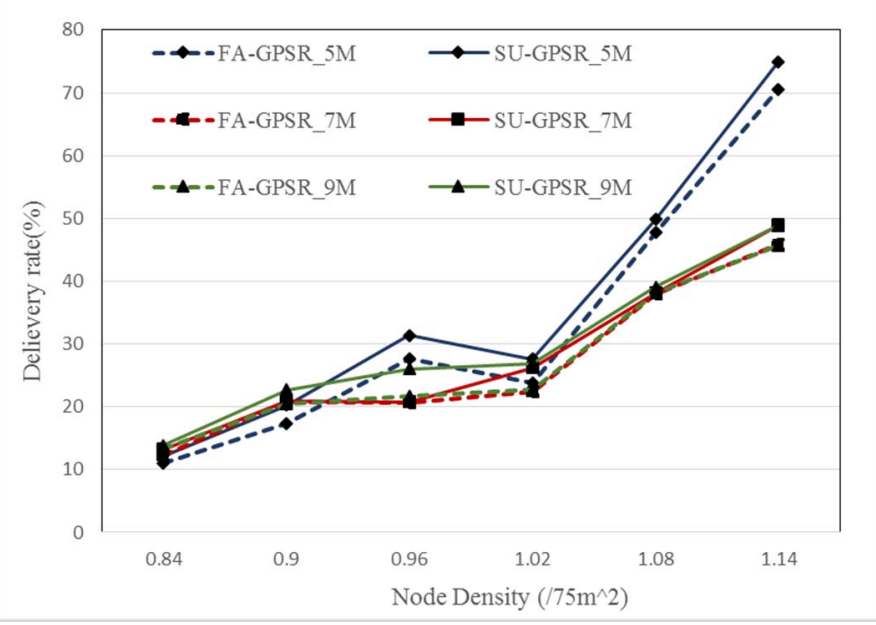

Fig. 7 Packet Delivery Sucessful Rate

The last performance indicator is illustrated in Fig. 5. The variation of successful packet delivery rate is very little between SU-GPSR and FA-GPSR. This means that by gaining more benefits, SU-GPSR doesn't sacrifice the data delivery.

\section{CONCLUSION}

This paper proposes a position-based routing scheme called Speed Up-Greedy Perimeter Stateless Routing Protocol for Wireless Sensor Networks (SU-GPSR). Two modes (Greedy and Speed Up) are designed in SU-GPSR to illustrate the packet forwarding status. Furthermore, the suggested next hop selection algorithm, not only applies the same criteria to both still and mobile nodes, but the energy for receiving and transmitting data is taken in to account during the calculation. The nodes with energy harvesting capability is covered in the algorithm as well. SU-GPSR is evaluated in OPNET simulation platform, comparing with two other geographical routing protocols: EGPRS and FA-GPSR. Overall SU-GPSR provide satisfied performance in reducing the end-to-end delay, less hop counts along the route and good successful packet delivery rate.

\section{REFERENCES}

[1] Kazem Sohraby, Daniel Minoli, and Taieb Znati. Wireless sensor networks: technology, protocols, and applications. John Wiley \& Sons, 2007.

[2] I. F. Akyildiz, W. Su, Y. Sankarasubramaniam, and E. Cayirci. Wireless
Sensor Networks: A survey. Computer. Network, 38(4):393-422, March 2002

[3] Ivan Stojmenovic, "Position-Based Routing in Ad Hoc Networks", IEEE Communications Magazine, July 2002, pp 128-134

[4] Y.C. Tseng, W.H. Liao, and S.L. Wu, "Mobile Ad Hoc Networks and Routing Protocols," Handbook of Wireless Networks and Mobile Computing, I. Stojmenovic, Ed., Wiley, 2002, pp. 371-92.

[5] G. G. Finn, "Routing and Addressing Problems in Large MetropolitanScale Internetworks," ISI res. rep. ISU/RR-87-180, Mar. 1987

[6] H. Takagi and L. Kleinrock, "Optimal Transmission Ranges for Randomly Distributed Packet Radio Terminals," IEEE Trans. Commun., vol. 32, no. 3, 1984, pp. 246-57.

[7] I. Stojmenovic and Xu Lin, "Loop-free Hybrid Single-Path/Flooding Routing Algorithms with Guaranteed Delivery for Wireless Networks," IEEE Trans. Parallel Dist. Sys., vol. 12, no. 10, 2001, pp. 1023-32.

[8] I. Stojmenovic and Xu Lin, "Power-Aware Localized Routing in Wireless Networks," IEEE Trans. Parallel Dist. Sys., vol. 12, no. 11, 2001, pp. 1122-33

[9] C. Yeh, "Variable-Radius Routing Protocols for High Throughput, Low Power, and Small Latency in Ad Hoc Wireless Networks," IEEE Int'l. Conf. WLANs and Home Nets., Dec. 2001

[10] P. Larsson, "Selection Diversity Forwarding in a Multihop Packet Radio Network with Fading Channel and Capture," Proc. ACM MobiHoc 2001, pp. 279-82.

[11] P. Bose et al., "Routing with Guaranteed Delivery in Ad Hoc Wireless Networks," 3rd Int'l. Wksp. Discrete Algorithms Methods Mobile Comp. Commun., Seattle, WA, Aug. 20, 1999, pp. 48-55; also in ACM/Kluwer WL Nets., vol. 7, no. 6, Nov. 2001, pp. 609-16

[12] Karp B , Kung H T. "GPSR : Greedy perimeter stateless routing for wireless networks", ACM/IEEE International Conference on Mobile Computing and Networking, Boston Massachusetts , USA , 2000 : 243-254

[13] T. Guoming, X. Yi, T. Daquan, and T. Jiuyang, "Divisional perimeter routing for gpsr based on left and right hand rules," in Computer Science and Network Technology (ICCSNT), 2011 International Conference on, vol. 2. IEEE, 2011, pp. 726-729

[14] W. Wei and Z. H. Yang, "Increasing packet delivery ratio in gpsr using buffer zone based greedy forwarding strategy," in Data Storage and Data Engineering (DSDE), 2010 International Conference on. IEEE, 2010, pp. 178-182.

[15] F. Granelli, G. Boato, D. Kliazovich, and G. Vernazza, "Enhanced gpsr routing in multi-hop vehicular communications through movement awareness," Communications Letters, IEEE, vol. 1 1, no. 10, pp. 781-783, 2007

[16] Y Alroqi , S Papanastasiou , E Peytchev, "Ferry-assisted greedy perimeter stateless routing protocol for mobile ad hoc networks (FAGPSR)", 2014 IEEE Symposium on Computers and Communication (ISCC) (2014), pp: 1-6

[17] Q. Xian, Y. Long, “An Enhanced Greedy Perimeter Stateless Routing Algorithm for Wireless Sensor Network", 2016 IEEE International Conference of Online Analysis and Computing Science (ICOACS 2016)

[18] J. Bai, M. Fan, J. Yang, Y. Sun, C. Phillips, "Smart Energy Harvesting Routing Protocol for WSN based E-Health Systems", 5th ACM MobiHoc Workshop on Pervasive Wireless Healthcare Proceedings (ACM MobileHealth'15). ACM, 2015. pp 23-28. 